\title{
Bilateral Carotid Endarterectomy Combined with Myocardial Revascularization During the Same Surgical Act
}

\author{
Leonardo Andrade Mulinari, André Luiz Tyszka, Arleto Zacarias Silva Jr, Fábio Binhara Navarro, \\ Roberto Gomes de Carvalho
}

Curitiba, PR - Brazil

The best surgical approach for the treatment of patients with severe cerebral artery disease and simultaneous serious coronary artery disease still remains controversial. In this report we present a case of a 72-year-old female patient admitted to the hospital with unstable angina. Triple coronary artery obstructive disease and severe bilateral carotid artery stenosis were diagnosed. A combined, simultaneous surgical procedure was performed. After total circulatory by-pass with a membrane oxygenator, the patient's body temperature was lowered to $32^{\circ} \mathrm{C}$. During the cool-down period, three proximal anastomoses of segments of autologous saphenous veins were performed in the ascending aorta. Immediately afterwards, bilateral carotid endarterectomy was performed, followed by three distal anastomoses to coronary arteries. The patient showed a satisfactory post-operative outcome. It was concluded that the combination of moderate hypothermia, hemodilution with appropriate hemodynamic control, as used in this patient, was an effective method of cerebral protection. The simultaneous approach of carotid endarterectomy and coronary artery by-pass surgery should be seen as a safe option for the treatment of this type of patient.

The involvement of both carotid arteries in significant stenosis has always been a challenge to the cardiovascular surgeon. The choice of the best surgical approach has been the cause of controversies in the literature, especially when an associated coronary lesion exists. Some centers follow the criterion of degree of seriousness, indicating that the first measure should be surgical treatment of the most endangered or symptomatic system, be it cardiac or cerebral ${ }^{1}$, but patients do exist for which such prioritization is difficult. Therefore, other centers in contrast advocate a simultaneous approach, which has several technical variations ${ }^{2-4}$.

Hospital Nossa Senhora das Graças - Curitiba

Mailing address: Leonardo Andrade Mulinari - Rua Silva Jardim, 3722/4 -

80240-021 - Curitiba, PR - Brazil

\section{Case Report}

A 72-year-old female with serious atherosclerotic systemic disease was hospitalized in the coronary unit for unstable angina pectoris, without neurological complaints. Her electrocardiogram revealed extensive anterior and inferior wall myocardial ischemia. Cineangiocardiography revealed serious coronary insufficiency with obstructive atherosclerotic damage to the anterior interventricular branch, the first left marginal branch and the right coronary artery; consensual indication for surgery was reached. Preoperative carotid Doppler echofluxometry demonstrated $95 \%$ stenosis of the lumen of the right internal carotid artery and $99 \%$ of that of the left. In view of the seriousness of the findings, simultaneous intervention in both carotids plus myocardial revascularization was the approach chosen.

The patient was monitored by pulse oxymetry, capnography, invasive arterial blood and central venous pressures, esophageal temperature and electrocardiography, as well pressures as by laboratory controls (blood gases, hematocrit and electrolytes). Following general anesthesia, the right and left carotid arteries were dissected and prepared. Exposure of the heart was made by median sternotomy. Following total heparinization, extracorporeal circulation was installed with cannulation of the aorta and the right atrium. While under extracorporeal circulation, the patient remained with a $20 \%$ hematocrit and strict hemodynamic control. During the hypothermia period, the proximal anastomoses of the safenous veins on the ascending aorta, which did not have calcified plaques, were performed. With the patient hemodynamically stabilized, hemodiluted and with a body temperature of $32^{\circ} \mathrm{C}$, endarterectomy of both internal carotid arteries was performed, first on the left side, then on the right, with resection of two large calcified and ulcerated plaques, without the need of a shunt. Following the main vascular period, cardiac surgery took its habitual course according to the center's routine, three coronary branches (anterior interventricular, first left marginal and posterior interventricular) being revascularized with the venous grafts.

The patient was directed to the Intensive Care Unit where she remained hemodynamically stable, not requiring the use of vasoactive drugs, and with a rather satisfactory evolution and no neurological or cardiovascular complica- 
tions. She was discharged from the Intensive Care Unit on the second day and from the hospital on the seventh day following the operation in clinically good condition.

\section{Discussion}

Acute myocardial infarction is the major cause of postoperative mortality in vascular surgery. Yet, stroke also remain a significant cause of morbidity and mortality (1 to $4 \%)^{5}$ after cardiac surgery. Although embolism from atherosclerotic plaques of the aorta and the heart, remains the cause of the majority of such accidents, vascular cerebral disease remains responsible for approximately $30 \%$ of them $\left(D^{\prime}\right.$ Agostino $\left.{ }^{6}\right)$. A more aggressive treatment in cases of combined diseases has shown the importance of diminishing the incidence of postoperative stroke, which is $(9.2 \%)^{7}$ higher in these patients. Carotid endarterectomy under deep hypothermia and full circulatory arrest remains an option for simultaneous treatment; it is known, however, that this procedure is associated with a greater risk of bleeding, as well as an inconveniently increased period of myocardial ischemia ${ }^{8}$.

We have used moderate hypothermia and hemodilution as auxiliary means to ensure cerebral protection; the great value of such measures has been pointed out ${ }^{9}$. Adequate hemodynamic control provided by intensive monitoring from the start of extracorporeal circulation until release from the Intensive Care Unit contributes to the good progress of patients. We believe that the surgical timing as presently used is also important because in our center it does not differ very much from that used in isolated myocardial revascularization; it is worth noting in this context, that to follow routine procedures always increases the surgeon's assurance and self-confidence. We had such assurance when performing the present complex procedure. The favorable evolution of the patient, furthermore, indicates the importance of a far-reaching preoperative evaluation, especially in patients with coronary disease and a past history of neurological events or peripheral vascular disease. It is our belief that the presently described approach is rather safe, easily reproduced and capable of bringing considerable benefits to patients.

Note added in proof: Since receiving notice of the acceptance for publication of this article, five other similar operations have been performed. All patients received myocardial revascularizations and bilateral endarterectomies of the carotid arteries and have shown a favorable evolution with the absence of cardiovascular and cerebral problems.

\section{Referências}

1. Johnson RG. Carotid endarterectomy and coronary artery bypass. The staged approach. In: The Thirty-First Postgraduate Program of The Society of Thoracic Surgeons. New Orleans, 1998: 21-23.

2. Vermeulen FEE, Hamerlijinck RPHM, Defauw JJAM, Ernst SMPG. Synchronous operation for ischemic cardiac and cerebrovascular disease: early results and long-term follow-up. Ann Thorac Surg 1992; 53: 381-90.

3. Kron IL. Combined carotid endarterectomy and coronary artery bypass grafting. In: The Thirty-First Postgraduate Program of The Society of Thoracic Surgeons. New Orleans, 1998: 19-20.

4. Pomè G, Passini L, Colucci V, et al. Combined surgical approach to coexistent carotid and coronary artery disease. J Cardiovasc Surg 1991; 32 787-93.

5. Mackey WC, Khabbaz K, Bojar R, O'Donnell TF. Simultaneous carotid endarte- rectomy and coronary bypass: Perioperative risk and long term survival. J Vasc Surg 1996; 24: 58-64.

6. D'Agostino RS, Svensson LG, Neumann DJ, et al. Screening carotid ultrasonography and risk factors for stroke in coronary artery surgery patients. Ann Thorac Surg 1996; 62:1714-23.

7. Rondino W, Panetta TF, Burak JH, et al. Combined carotid endarterectomy, innominate artery reconstruction, and coronary artery bypass grafting: Case Report. J Vasc Surg 1996; 24: 1017-21.

8. Weiss SJ, Sutter FP, Shannon TO, Goldman SC. Combined cardiac operation and carotid endarterectomy during aortic cross-clamping. Ann Thorac Surg 1992; 53: 813-9.

9. Wareing TH, Davila-Roman VG, Daily BB, et al. Strategy for reduction of stroke incidence in cardiac surgical patients. Ann Thorac Surg 1993; 55: 1400-8. 